\title{
Clinical Diagnosis of Bordetella Pertussis Infection: A Systematic Review
}

\author{
Mark H. Ebell, MD, MS, Christian Marchello, MS, MT, and Maria Callahan
}

Background: Bordetella pertussis (BP) is a common cause of prolonged cough. Our objective was to perform an updated systematic review of the clinical diagnosis of BP without restriction by patient age.

Methods: We identified prospective cohort studies of patients with cough or suspected pertussis and assessed study quality using QUADAS-2. We performed bivariate meta-analysis to calculate summary estimates of accuracy and created summary receiver operating characteristic curves to explore heterogeneity by vaccination status and age.

Results: Of 381 studies initially identified, 22 met our inclusion criteria, of which 14 had a low risk of bias. The overall clinical impression was the most accurate predictor of BP (positive likelihood ratio $[\mathrm{LR}+], 3.3$; negative likelihood ratio [LR-], 0.63). The presence of whooping cough $(\mathrm{LR}+, 2.1)$ and posttussive vomiting $(\mathrm{LR}+, 1.7)$ somewhat increased the likelihood of $\mathrm{BP}$, whereas the absence of paroxysmal cough $(L R-, 0.58)$ and the absence of sputum $(L R-, 0.63)$ decreased it. Whooping cough and posttussive vomiting have lower sensitivity in adults. Clinical criteria defined by the Centers for Disease Control and Prevention were sensitive (0.90) but nonspecific. Typical signs and symptoms of BP may be more sensitive but less specific in vaccinated patients.

Conclusions: The clinician's overall impression was the most accurate way to determine the likelihood of BP infection when a patient initially presented. Clinical decision rules that combine signs, symptoms, and point-of-care tests have not yet been developed or validated.(J Am Board Fam Med 2017;30:308-319.)

Keywords: Bordetella Pertussis, Vaccination, Vomiting, Whooping Cough

Pertussis is an acute respiratory illness caused by the Bordetella pertussis (BP) bacterium. Initial symptoms are usually relatively mild, and can include cough, sneezing, coryza, and slight fever. The disease then progresses to produce more severe, paroxysmal coughing fits. During these episodes, the characteristic "whooping" may be heard as the patient tries to inhale through narrowed upper airways and the larynx. Posttussive vomiting, cyanosis, and apnea can occur as a result of the severity of these episodes. ${ }^{1}$ A recent systematic review of the

This article was externally peer reviewed.

Submitted 20 October 2016; revised 13 January 2017; accepted 13 January 2017.

From the Department of Epidemiology, University of Georgia, Athens (MHE, CM, MC).

Funding: none.

Conflict of interest: none declared.

Corresponding author: Mark H. Ebell, MD, MS, University of Georgia, Health Sciences Campus, 233 Miller Hall, Athens, GA 30602 (E-mail: ebell@uga.edu). prevalence of $\mathrm{BP}$ infection in patients with prolonged cough found that the overall prevalence of $\mathrm{BP}$ infection among primary care patients with prolonged cough (typically $>1$ week) was $12.4 \%$; it was $18 \%$ in studies of children-much higher than suggested by laboratory-based surveillance reports. ${ }^{2}$ The recent large increase in pertussis cases in the United States has been described by the Centers for Disease Control and Prevention (CDC) as an epidemic. $^{3}$

For infants and young children, complications from pertussis may be life-threatening, and hospitalization may be warranted. Vaccination against $\mathrm{BP}$ is effective at preventing the spread of infection and is recommended for infants, children, and adults. ${ }^{4,5}$ Though older children and adults are unlikely to experience severe complications from pertussis, they can act as vectors of infection when in contact with those who are more vulnerable, including unvaccinated or incompletely vaccinated infants and children. ${ }^{6,7}$ 
CDC guidelines recommend a macrolide antibiotic (azithromycin, clarithromycin, or erythromycin), or, as an alternative, trimethoprim-sulfamethoxazole, for patients diagnosed with pertussis. Ideally, treatment should begin within 1 to 2 weeks of the onset of symptoms. ${ }^{8}$ However, recognition of patients with pertussis is challenging, as many of their symptoms overlap with those of viral acute respiratory tract infections, and the recommended diagnostic test (polymerase chain reaction [PCR]) typically takes several days. Thus, signs and symptoms may be able to help identify patients at high risk for pertussis, for whom testing should be prioritized. We identified only a single previous systematic review of clinical diagnosis, which excluded studies of young children and studies using culture as a reference standard. ${ }^{1}$ A number of potentially relevant studies have also been published since its search was completed in 2010. The purpose of this meta-analysis is to perform a comprehensive systematic review and meta-analysis of the accuracy of signs and symptoms for the diagnosis of pertussis in patients of all ages.

\section{Methods}

\section{Literature Search}

We systematically reviewed studies published in MEDLINE using the following search strategy:

"pertussis"[tiab] OR “whooping cough"[tiab]) AND ("medical history taking"[MeSH Terms] OR "physical examination” [MeSH Terms] OR "signs and symptoms" OR "symptoms and signs" OR "symptom" [Title/Abstract] OR "history and physical” OR "physical examination" OR "physical examination" OR "clinical diagnosis" OR "clinical examination" OR "post-tussive" OR "posttussive" OR "vomiting" OR "c-reactive protein" OR "CRP” OR "sedimentation rate" OR "sed rate" or "WESR" or "procalcitonin" OR "white blood cell count" OR "complete blood count" OR "WBC" OR "CBC"

We did not restrict the search by language or date of publication. In addition, we searched the reference lists of included studies for additional studies not initially identified in our MEDLINE search.

\section{Inclusion and Exclusion Criteria}

We included prospective cohort studies of patients presenting with acute cough, prolonged cough, or clinically suspected pertussis if all patients received a reference standard test consisting of PCR, culture, or serology, and if studies provided adequate data to calculate either the sensitivity or specificity of the examined symptoms. We excluded studies if they used a case-control design (which overestimates treatment accuracy) ${ }^{9}$; if sign and symptom data were collected retrospectively or after the initial patient contact; if the study was performed in a specialized population (eg, immunocompromised patients); if data were gathered only after a positive test had been obtained; or if the study had fewer than 25 patients. ${ }^{10,11}$ Since outbreak investigations typically gather symptom data retrospectively, and often by telephone, they were not included. Our goal was to identify high-quality studies that gathered clinical data at the time the patient presented, masked to the results of the reference standard test. No age restriction or restriction by immunization status was used.

\section{Data Abstraction}

Each abstract was reviewed by 2 investigators (MC and $\mathrm{CM}$ ) for possible inclusion, and the full text of any study that potentially fit the inclusion criteria was reviewed in detail. Two investigators (MC and $\mathrm{CM}$ ), working in parallel, abstracted data on study characteristics, study quality, and the accuracy of signs and symptoms. Any discrepancies were resolved by consensus after discussion, involving the third investigator (ME) if necessary.

\section{Quality Assessment}

The studies were evaluated for potential bias using the QUADAS-2 framework, which assesses patient selection, index test, reference standard used, and patient flow. ${ }^{12}$ The reference standard was considered to be at low risk of bias if PCR, culture, paired serology, or some combination of those was used to evaluate all patients in the study, whereas single serology was considered to have a high risk of bias. Assessments were conducted in parallel, such that each study was examined by at least 2 of the investigators, and disagreements were resolved by consensus after discussion.

\section{Statistical Analysis}

We performed bivariate meta-analysis using the mada and metafor procedures in $\mathrm{R}$, version 3.2.2. We calculated summary estimates and $95 \%$ confidence intervals for sensitivity, specificity, positive 
likelihood ratios $(\mathrm{LR}+)$, negative likelihood ratios (LR-), and diagnostic odds ratios (DORs) using the Reitsma method from the mada procedure. This method can only calculate summary estimates when both sensitivity and specificity are available. Some of the included studies did not report specificity. In these situations, the summary estimates of sensitivity were calculated using a random effects model of raw proportions using the metafor procedure. The raw proportion was the sensitivity for each symptom, calculated by the number of true positives divided by true positive plus false negative $(\mathrm{TP} /[\mathrm{TP}+\mathrm{FN}])$. Two variables (headache and wheezing) had positive likelihood ratios $<1.0$, so we report the accuracy of the absence of these signs or symptoms. We used summary receiver operating characteristic (ROC) curves to explore heterogeneity and plot $95 \%$ confidence intervals for the summary estimates of accuracy.

\section{Results}

The results of the search process are summarized in the Preferred Reporting Items for Systematic Re-

Figure 1. Preferred Reporting Items for Systematic Reviews and Meta-analyses flow diagram. Only the first identified reason for excluding an article is listed. In some cases $>1$ reason was identified.

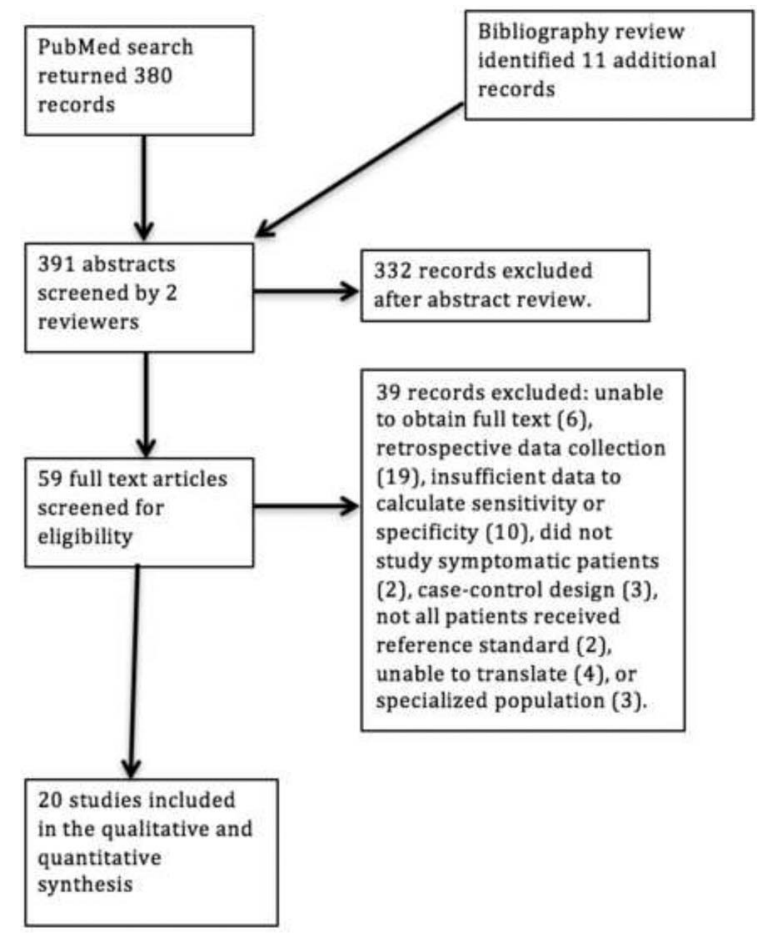

views and Meta-analyses diagram (Figure 1). The initial MEDLINE search identified 381 studies, of which 17 were included; a review of the reference lists of included studies identified 5 more. Overall, 22 studies with a total of 15,909 patients met the inclusion and exclusion criteria. Their characteristics are summarized in Table 1. Twelve were set in Europe, 2 in Korea, and the remainder each in 1 of 8 countries in Australasia, Africa, or the Americas. Study size ranged from 32 to 3,629 patients. Nineteen studies used PCR, culture, or paired serology as the reference standard; only 3 studies used a single serology as the reference standard. Four studied adults only, 8 studied children only, and 10 studied both adults and children.

\section{Quality Assessment}

The quality assessment is summarized in Table 2, using the QUADAS-2 framework. Overall, we judged 14 studies to be at low risk of bias, 4 at moderate risk of bias, and 4 at high risk of bias.

\section{Accuracy of Signs and Symptoms}

The accuracy of individual signs and symptoms (reported by at least 3 studies) is summarized in Table 3 (full results by individual study are available in the online Appendix). The overall clinical assessment by the physician was the most helpful single piece of information at ruling in pertussis when positive, with an LR+ of 3.3, an LR- of 0.63 , and a DOR of 5.3. While the overall impression of a physician was quite specific (0.85), it lacked sensitivity (0.47) and would therefore fail to identify more than half of patients with pertussis.

The following individual signs and symptoms were significantly associated with pertussis (LR+ and LR - both were significantly different from 1.0): whooping cough (overall and in children only), posttussive vomiting, paroxysmal cough, sputum, sleep disturbed by cough, and absence of headache. However, the likelihood ratios were all between 2.1 and 0.58 , indicating little diagnostic value.

While paroxysmal cough was the most sensitive single finding, it lacked specificity $(0.35)$ because it occurs in many other respiratory infections. The 3 signs or symptoms that were best at ruling out pertussis when absent or negative were the overall clinical assessment by a physician (LR-, 0.57), the absence of paroxysmal cough ( $\mathrm{LR}-, 0.58)$, and the absence of sputum production (LR-, 0.63). Still, 


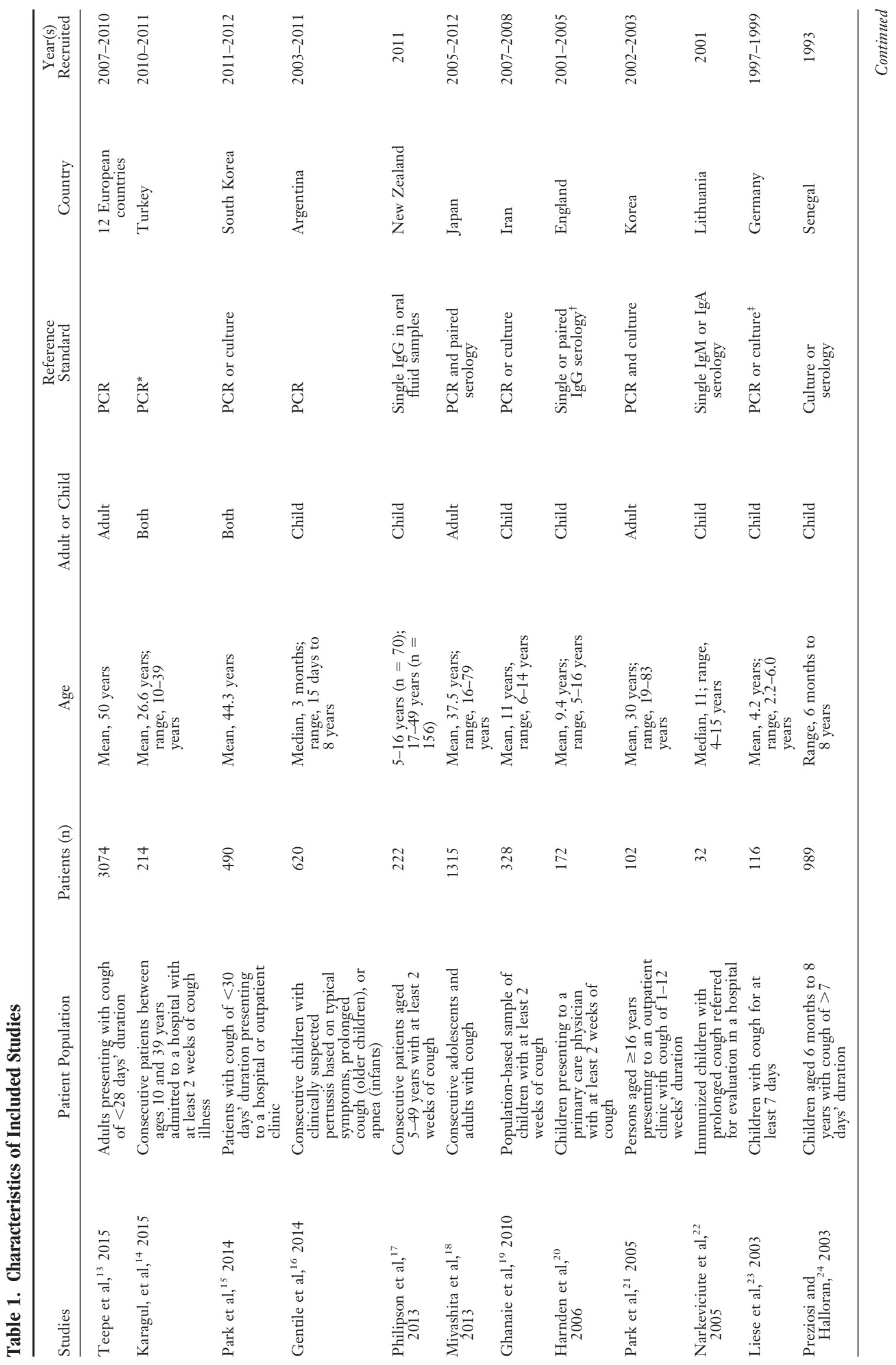




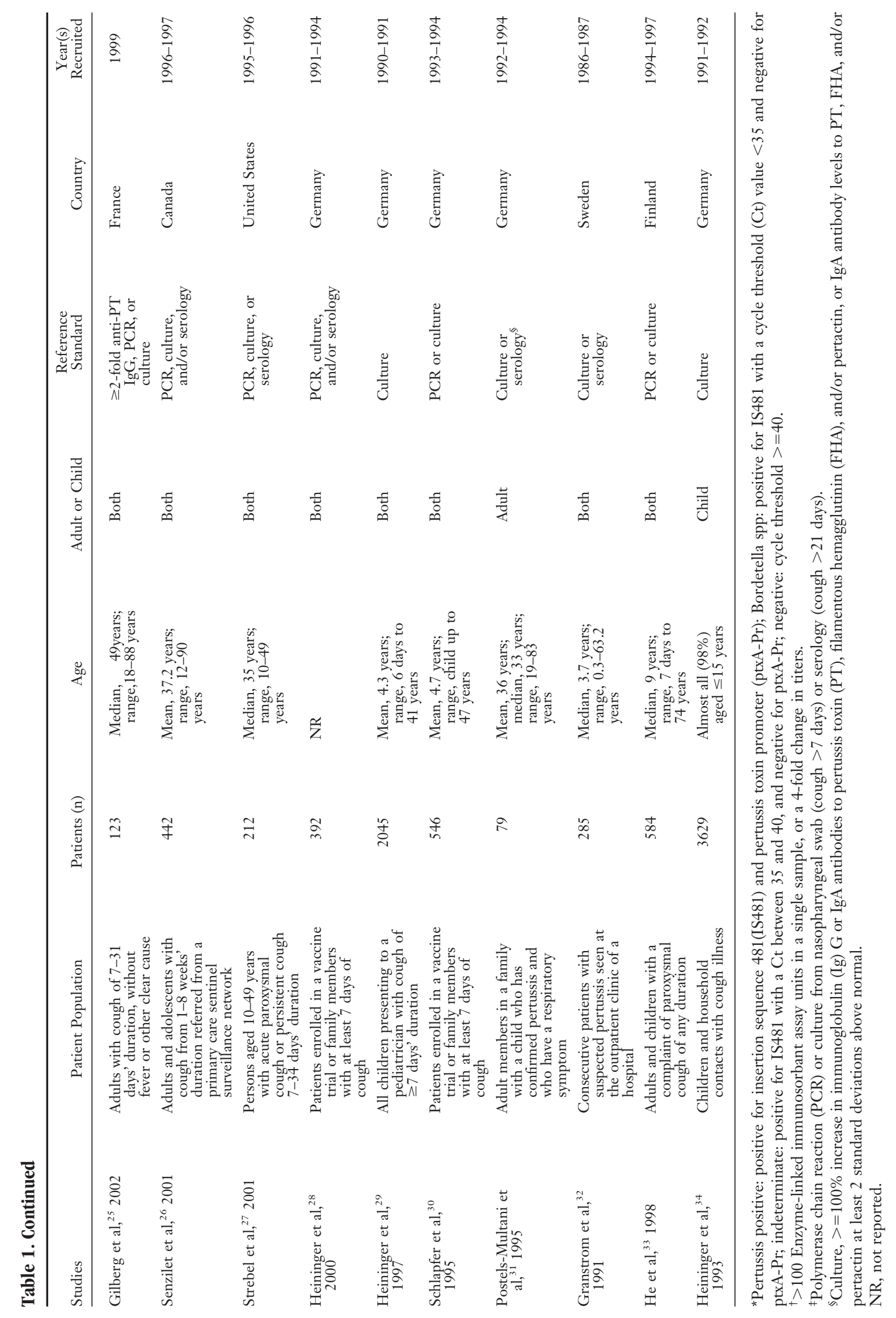




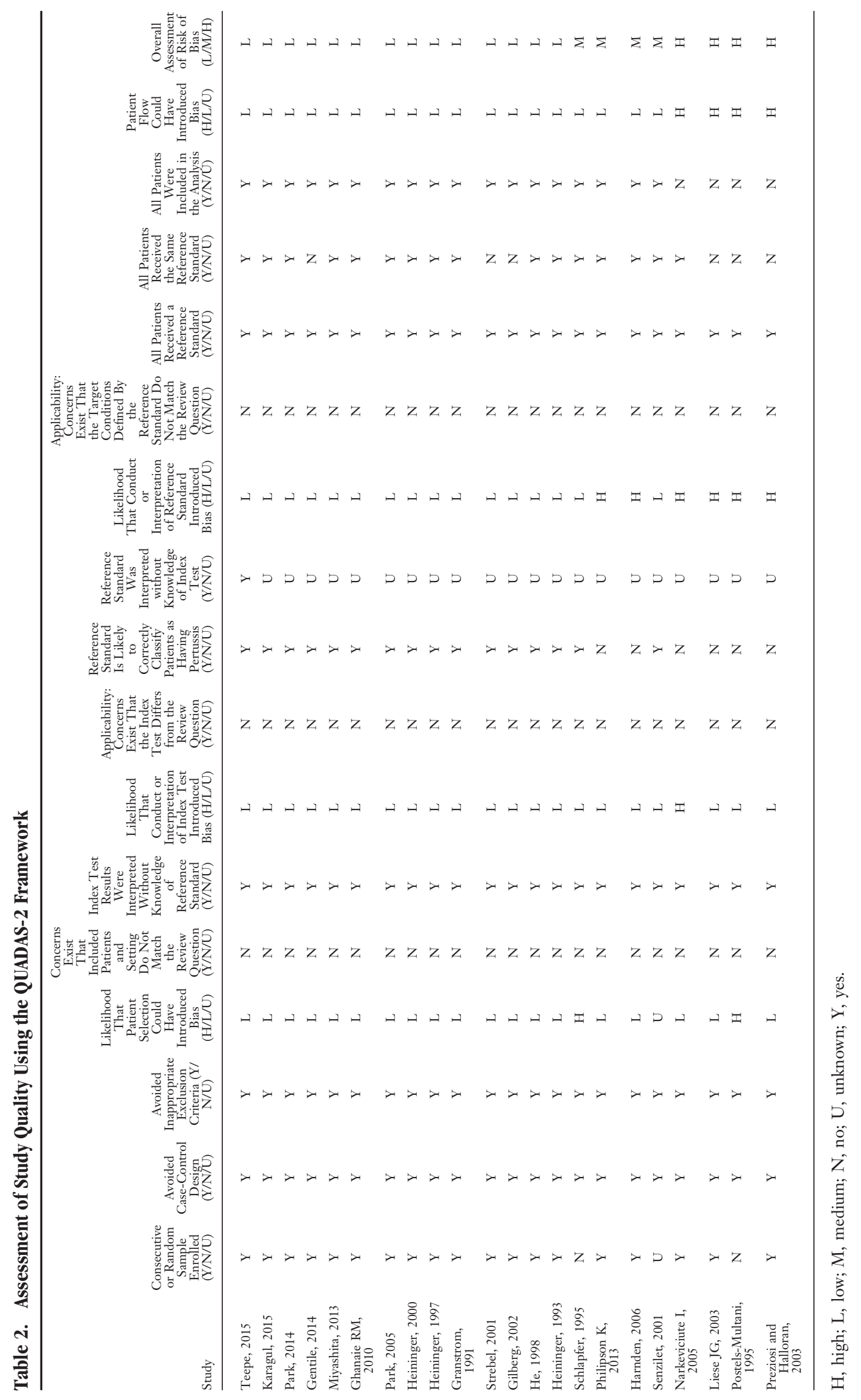




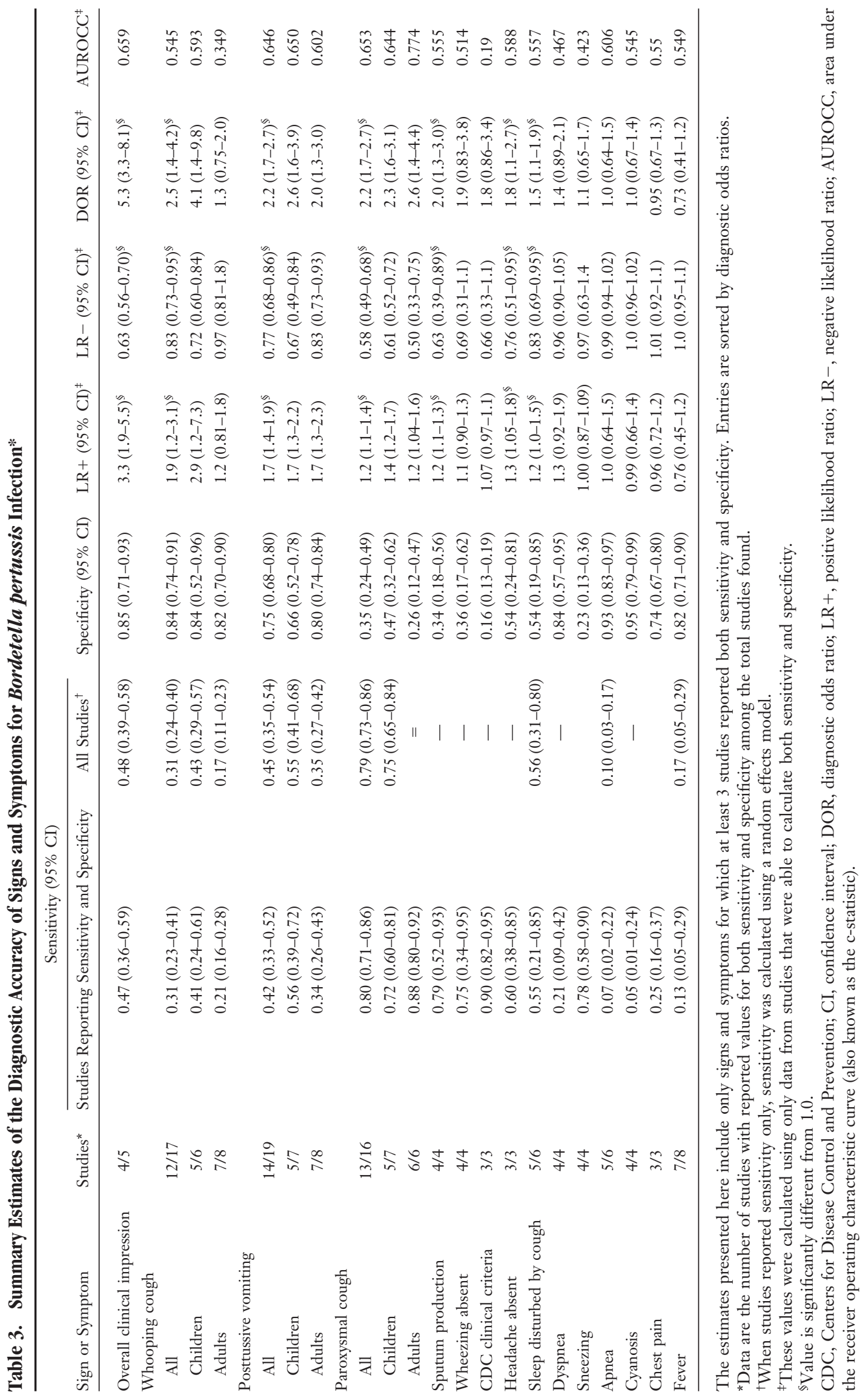


these LR - values are quite close to 1.0 and are therefore only minimally helpful.

Because prolonged cough was often part of the inclusion criteria, it was not reported by most studies as a clinical variable. When it was reported, studies generally indicated the overall duration of cough for the episode of illness, rather than the duration of cough at the time the patient initially presented to a clinician. Two studies did report the likelihood of pertussis for patients with cough for $>2$ weeks at presentation, compared with a shorter duration. One was a large, contemporary European multicenter study that enrolled 3074 patients, of whom 93 had pertussis by PCR. Pertussis was more likely among patients with cough for $>2$ weeks, but the sensitivity was only $27 \%$ and specificity $82 \%$ $(\mathrm{LR}+, 1.5 ; \mathrm{LR}-, 0.89) .{ }^{13}$ An older study also reported data for this variable, but similarly did not find it to be a helpful predictor of pertussis $(\mathrm{LR}+$, 1.1; LR - , 0.98). ${ }^{30}$

We were able to evaluate separately for adults and children the accuracy for 3 key symptoms: whooping cough, posttussive vomiting, and paroxysmal cough. Both whooping cough (41\% vs 20\%) and posttussive vomiting (56\% vs $34 \%$ ) were more sensitive in children, although the specificity was similar. The LR + for whooping cough was 4.1 in children and only 1.4 in adults.

No studies of a clinical decision rule (CDR) combining data for several signs and symptoms met our inclusion criteria. One study used the combination of symptoms and the local prevalence of pertussis for the week in which a sample was drawn to predict the likelihood of infection. However, it gathered data retrospectively and has not been prospectively or externally validated. ${ }^{35}$ The CDC has defined the clinical case for pertussis as cough for $\geq 14$ days plus at least 1 other typical symptom (paroxysms of cough, inspiratory whoop, or posttussive vomiting). Three studies reported the accuracy of the CDC criteria, with summary estimates of accuracy at $90 \%$ sensitivity, only $16 \%$ specificity, LR + of 1.1, and LR - of $0.66 .^{19,25,27}$

\section{Summary ROC Curves}

Summary ROC curves for key signs and symptoms are shown in Figure 2A through D, stratified by age, where possible. The ROC curve for the overall clinical impression (Figure 2A) shows good consistency and a fairly narrow confidence interval around the summary estimate of accuracy. As noted earlier, whooping cough (Figure 2B) and posttussive vomiting (Figure $2 \mathrm{C}$ ) were both more sensitive in children than in adults. Apnea and cyanosis (Figure 2D) are plotted together because they had very similar sensitivity and specificity values. Of 4 studies reporting data for cyanosis, 3 found that it was highly specific $(>0.95)$ but lacked sensitivity.

\section{Vaccination Status}

Summary ROC curves comparing populations that have high rates of vaccination with those that have low rates are shown in Figure 3A through D. Given the small number of studies in these comparisons, the ability to draw inferences is limited, and the data should be interpreted cautiously. The observed pattern based on these limited data is that in a vaccinated population, typical signs and symptoms of pertussis are more sensitive but less specific than those in an unvaccinated population.

\section{Discussion}

We have reviewed the best available evidence regarding the clinical diagnosis of BP infection in adults and children. The overall quality of the included studies, aided by the strict inclusion criteria of our systematic review, was good, with over half at low risk of bias. Unfortunately, we must conclude that individual signs and symptoms are of limited value, with none having an $\mathrm{LR}+>2.1$ or an LR $-<0.5$. The exception is the overall clinical impression, which had an LR+ of 3.3 and an LRof 0.63 . While the overall clinical impression has excellent specificity, it lacks sensitivity and would fail to identify half of the patients with pertussis. However, it clearly suggests that when a clinician has a strong clinical suspicion for pertussis, he or she should trust that suspicion and order confirmatory testing. Of course, the positive predictive value depends on the prevalence of pertussis. For example, it is $15 \%$ when the prevalence is $5 \%, 27 \%$ when the prevalence is $10 \%$, and $45 \%$ when the prevalence is $20 \%$.

Our overall summary estimates of accuracy were similar to those of a previous systematic review. ${ }^{1}$ However, we identified a number of additional studies, including 7 published since that review, and were able to stratify our results by adult versus child and vaccinated versus unvaccinated populations. Two symptoms, whooping cough and posttussive vomiting, were more sensitive in children 
Figure 2. Summary receiver operating characteristic curves for overall impression (A), whooping cough (B), posttussive vomiting (C), and apnea or cyanosis (D), stratified by age.

A

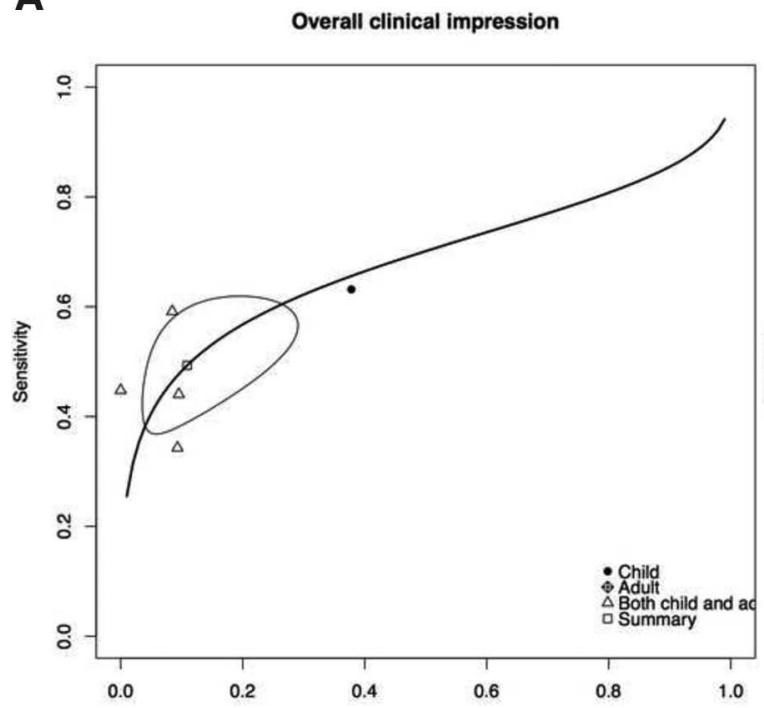

C

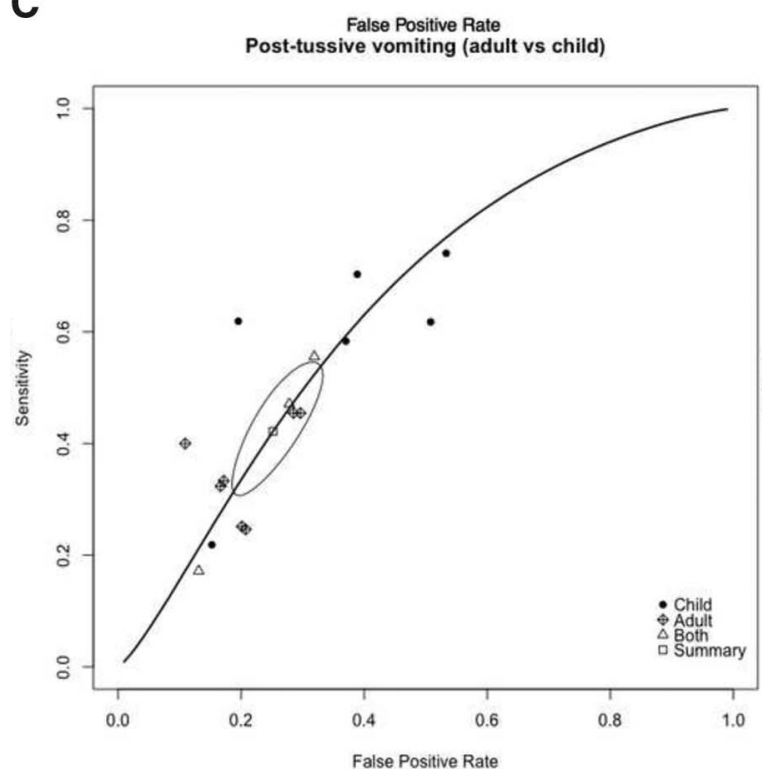

than in adults. This may be because of physiologic differences in airway size in children versus in adults; aerophagia, which is more common in children than in adults; and possibly a lower threshold for vomiting in children than in adults. Whooping and posttussive vomiting are therefore less diagnostically useful in adults, and the absence of these symptoms does not rule out pertussis in this group.

Based on a review of the ROC curves, it seems that typical signs and symptoms of pertussis are more sensitive but less specific in a vaccinated pop-
B

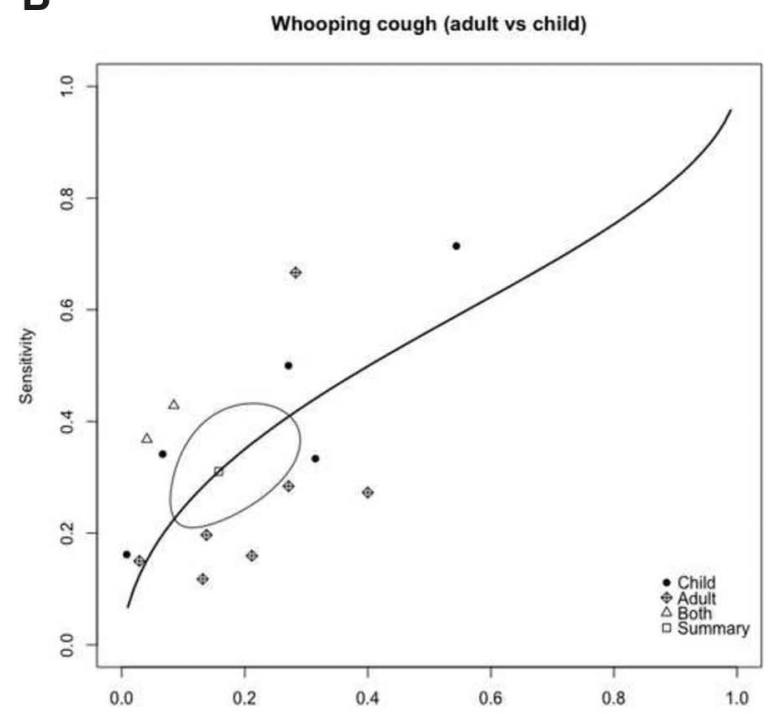

D

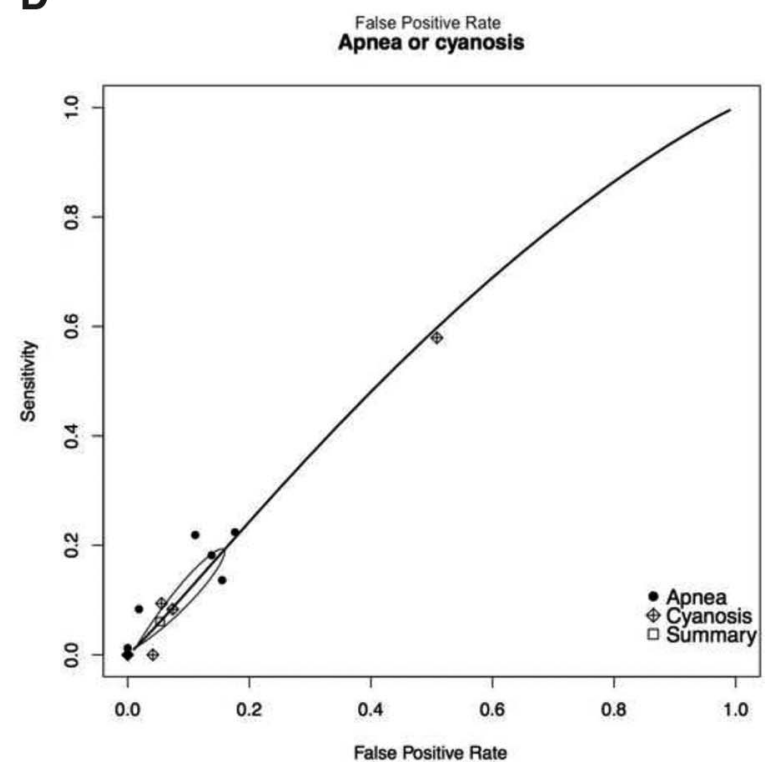

ulation than in an unvaccinated population. More sensitive signs and symptoms may have their greatest value in ruling out pertussis when negative. However, the small number of studies makes it difficult to draw conclusions, highlighting the need for studies of clinical diagnoses among contemporary vaccinated populations. These possible differences in the presentation between vaccinated and unvaccinated populations makes it important that we perform contemporary, well-designed, and adequately powered studies of signs, symptoms, and CDRs, as older data may no longer apply to the 
Figure 3. Summary receiver operating characteristic curves for overall impression (A), whooping cough (B), paroxysmal cough (C), and posttussive vomiting (D), stratified by vaccination status.

A

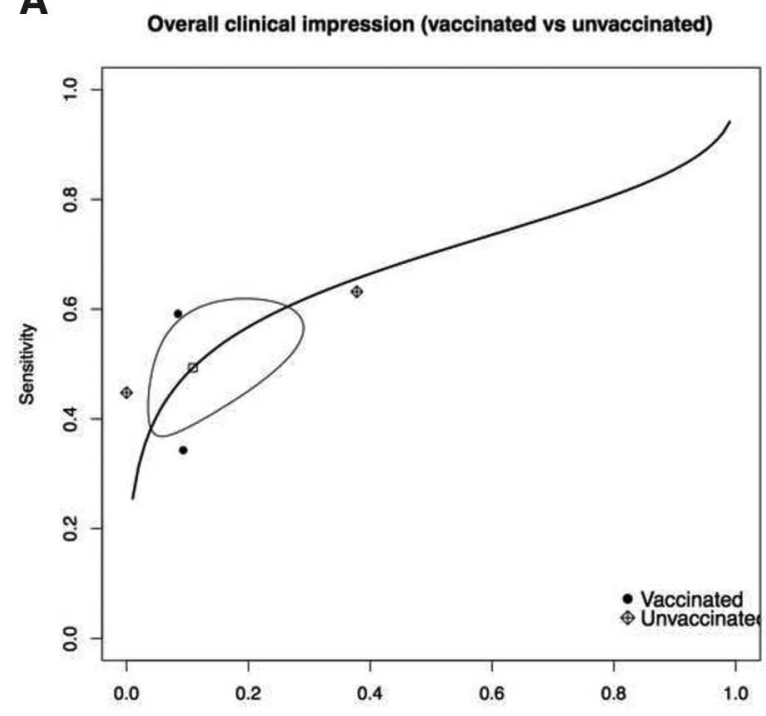

C

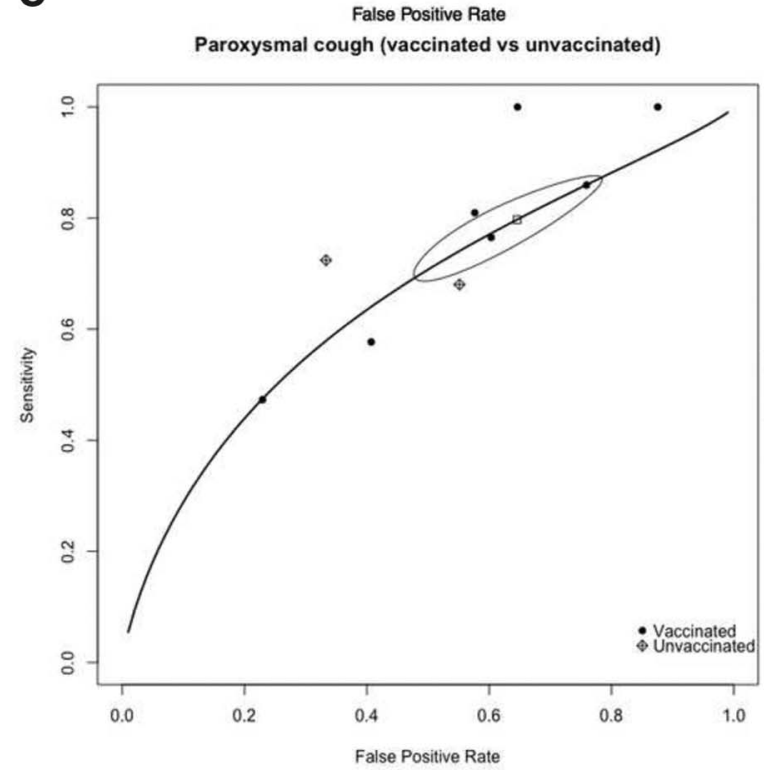

many parts of the world where $>95 \%$ of the population is vaccinated.

Our systematic review had several limitations. Data regarding the duration of a symptom or combination of symptoms and their association with the diagnosis were limited. The area under the summary ROC curves calculated by the mada procedure were somewhat unreliable because of sparse data and clustering of data in 1 part of many of the curves. The small number of studies for comparing vaccinated with unvaccinated populations limited out ability to draw conclusions, and some studies
B

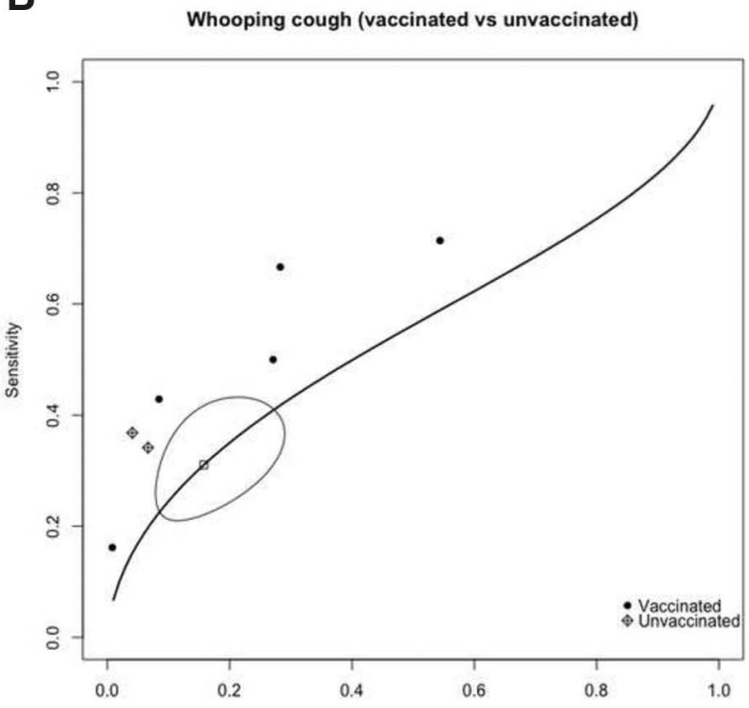

D

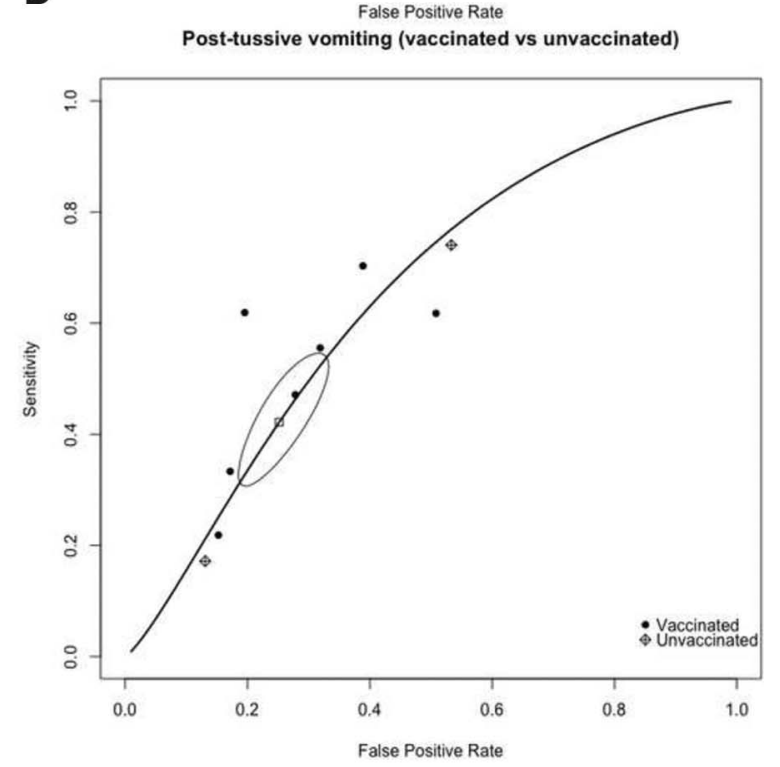

were quite old. Finally, considerable heterogeneity exists for common symptoms of pertussis, such as whooping cough and paroxysmal cough. This could be a result of differences in how the question was asked or differences in the population (adult vs child, vaccinated vs unvaccinated).

An important gap in the literature is the lack of validated CDRs. CDRs have been useful in other acute respiratory infections such as pharyngitis ${ }^{36}$ and pneumonia. ${ }^{37}$ They typically classify patients as low, moderate, and high risk; these categories correspond to patients in whom disease is ruled out, 
further testing is needed, or disease is ruled in. ${ }^{38,39}$ We identified only 1 CDR for pertussis; it is for infants and incorporates surveillance data but has not been prospectively validated. ${ }^{35}$ The CDC's definition of a clinical case could be considered a kind of CDR; however, it is sensitive but very nonspecific (16\%). This reflects the fact that it is a clinical case definition intended for use in outbreaks, with the knowledge that more specific confirmatory testing with culture or PCR would be available.

CDRs for other conditions have sometimes incorporated point-of-care tests such as C-reactive protein or procalcitonin ${ }^{40,41}$; this is a promising area for further research in the diagnosis of pertussis. Another, as demonstrated by Fine and colleagues, ${ }^{35,42}$ is the incorporation of surveillance data or novel approaches like using Google searches to predict the prevalence of pertussis.

To see this article online, please go to: http://jabfm.org/content/ 30/3/308.full.

\section{References}

1. Cornia PB, Hersh AL, Lipsky BA, Newman TB, Gonzales R. Does this coughing adolescent or adult patient have pertussis? JAMA 2010;304:890-6.

2. Marchello C, Dale AP, Thai TN, Han DS, Ebell MH. Prevalence of atypical pathogens in patients with cough and community-acquired pneumonia: a meta-analysis. Ann Fam Med 2016;14:552-66.

3. Winter K, Glaser C, Watt J, Harriman K, Centers for Disease C, Prevention. Pertussis epidemic-California, 2014. MMWR Morb Mortal Wkly Rep 2014; 63:1129-32.

4. Koepke R, Eickhoff JC, Ayele RA, et al. Estimating the effectiveness of tetanus-diphtheria-acellular pertussis vaccine (Tdap) for preventing pertussis: evidence of rapidly waning immunity and difference in effectiveness by Tdap brand. J Infect Dis 2014;210: 942-53.

5. Klein NP, Bartlett J, Fireman B, Rowhani-Rahbar A, Baxter R. Comparative effectiveness of acellular versus whole-cell pertussis vaccines in teenagers. Pediatrics 2013;131:e1716-22.

6. Bisgard KM, Pascual FB, Ehresmann KR, et al. Infant pertussis. Pediatr Infect Dis J 2004;23:985-9.

7. Wendelboe AM, Njamkepo E, Bourillon A, et al. Transmission of Bordetella pertussis to young infants. Pediatr Infect Dis J 2007;26:293-9.

8. Centers for Disease Control and Prevention. Pertussis (whooping cough): treatment. Available from: http://www.cdc.gov/pertussis/clinical/treatment. html. Accessed July 13, 2016.
9. Lijmer JG, Bossuyt PM, Heisterkamp SH. Exploring sources of heterogeneity in systematic reviews of diagnostic tests. Stat Med 2002;21:1525-37.

10. Dechartres A, Trinquart L, Boutron I, Ravaud P. Influence of trial sample size on treatment effect estimates: meta-epidemiological study. BMJ 2013; 346:f2304.

11. Nuesch E, Trelle S, Reichenbach S, et al. Small study effects in meta-analyses of osteoarthritis trials: meta-epidemiological study. BMJ 2010;341:c3515.

12. Whiting PF, Rutjes AW, Westwood ME, et al. QUADAS-2: a revised tool for the quality assessment of diagnostic accuracy studies. Ann Intern Med 2011;155:529-36.

13. Teepe J, Broekhuizen BD, Ieven M, et al. Prevalence, diagnosis, and disease course of pertussis in adults with acute cough: a prospective, observational study in primary care. Br J Gen Pract 2015;65: e662-7.

14. Karagul A, Ogunc D, Midilli K, et al. Epidemiology of pertussis in adolescents and adults in Turkey. Epidemiol Infect 2015;143:2613-8.

15. Park S, Lee SH, Seo KH, et al. Epidemiological aspects of pertussis among adults and adolescents in a Korean outpatient setting: a multicenter, PCR-based study. J Korean Med Sci 2014;29: 1232-9.

16. Gentile A, Romanin VS, Juarez Mdel V, Lucion MF, Marques Mde L, Mistchenko AS. Epidemiology of Bordetella pertussis in a children's hospital. Arch Argent Pediatr 2014;112:26-32.

17. Philipson K, Goodyear-Smith F, Grant CC, Chong A, Turner N, Stewart J. When is acute persistent cough in school-age children and adults whooping cough? A prospective case series study. Br J Gen Pract 2013;63:e573-9.

18. Miyashita N, Akaike H, Teranishi H, et al. Diagnostic value of symptoms and laboratory data for pertussis in adolescent and adult patients. BMC Infect Dis $2013 ; 13: 129$.

19. Ghanaie RM, Karimi A, Sadeghi H, et al. Sensitivity and specificity of the World Health Organization pertussis clinical case definition. Int $\mathrm{J}$ Infect Dis 2010;14:e1072-5.

20. Harnden A, Grant C, Harrison T, et al. Whooping cough in school age children with persistent cough: prospective cohort study in primary care. BMJ 2006; 333:174-7.

21. Park WB, Park SW, Kim HB, Kim EC, Oh M, Choe $\mathrm{KW}$. Pertussis in adults with persistent cough in South Korea. Eur J Clin Microbiol Infect Dis 2005; 24:156-8.

22. Narkeviciute I, Kavaliunaite E, Bernatoniene G, Eidukevicius R. Clinical presentation of pertussis in fully immunized children in Lithuania. BMC Infect Dis 2005;5:40.

23. Liese JG, Renner C, Stojanov S, Belohradsky BH; Munich Vaccine Study Group. Clinical and epide- 
miological picture of $\mathrm{B}$ pertussis and $\mathrm{B}$ parapertussis infections after introduction of acellular pertussis vaccines. Arch Dis Child 2003;88:684-7.

24. Preziosi MP, Halloran ME. Effects of pertussis vaccination on disease: vaccine efficacy in reducing clinical severity. Clin Infect Dis 2003;37:772-9.

25. Gilberg S, Njamkepo E, Du Chatelet IP, et al. Evidence of Bordetella pertussis infection in adults presenting with persistent cough in a French area with very high whole-cell vaccine coverage. J Infect Dis 2002;186:415-8.

26. Senzilet LD, Halperin SA, Spika JS, et al. Pertussis is a frequent cause of prolonged cough illness in adults and adolescents. Clin Infect Dis 2001;32: 1691-7.

27. Strebel P, Nordin J, Edwards K, et al. Populationbased incidence of pertussis among adolescents and adults, Minnesota, 1995-1996. J Infect Dis 2001; 183:1353-9.

28. Heininger U, Schmidt-Schlapfer G, Cherry JD, Stehr K. Clinical validation of a polymerase chain reaction assay for the diagnosis of pertussis by comparison with serology, culture, and symptoms during a large pertussis vaccine efficacy trial. Pediatrics 2000;105:E31.

29. Heininger U, Klich K, Stehr K, Cherry JD. Clinical findings in Bordetella pertussis infections: results of a prospective multicenter surveillance study. Pediatrics 1997;100:E10.

30. Schlapfer G, Cherry JD, Heininger U, et al. Polymerase chain reaction identification of Bordetella pertussis infections in vaccinees and family members in a pertussis vaccine efficacy trial in Germany. Pediatr Infect Dis J 1995;14:209-14.

31. Postels-Multani S, Schmitt HJ, Wirsing von Konig CH, Bock HL, Bogaerts H. Symptoms and complications of pertussis in adults. Infection $1995 ; 23: 139-42$.

32. Granstrom G, Wretlind B, Granstrom M. Diagnostic value of clinical and bacteriological findings in pertussis. J Infect 1991;22:17-26.
33. He Q, Viljanen MK, Arvilommi H, Aittanen B, Mertsola J. Whooping cough caused by Bordetella pertussis and Bordetella parapertussis in an immunized population. JAMA 1998;280:635-7.

34. Heininger U, Cherry JD, Eckhardt T, Lorenz C, Christenson P, Stehr K. Clinical and laboratory diagnosis of pertussis in the regions of a large vaccine efficacy trial in Germany. Pediatr Infect Dis J 1993; 12:504-9.

35. Fine AM, Reis BY, Nigrovic LE, et al. Use of population health data to refine diagnostic decisionmaking for pertussis. J Am Med Inform Assoc 2010; 17:85-90.

36. McIsaac WJ, Goel V, To T, Low DE. The validity of a sore throat score in family practice. CMAJ 2000; 163:811-5.

37. van Vugt SF, Broekhuizen BD, Zuithoff NP, et al. Validity of a clinical model to predict influenza in patients presenting with symptoms of lower respiratory tract infection in primary care. Fam Pract 2015; 32:408-14.

38. Ebell M. AHRQ white paper: use of clinical decision rules for point-of-care decision support. Med Decis Making 2010;30:712-21.

39. Ebell MH, Locatelli I, Senn N. A novel approach to the determination of clinical decision thresholds. Evid Based Med 2015;20:41-7.

40. van Vugt SF, Broekhuizen BD, Lammens C, et al. Use of serum $\mathrm{C}$ reactive protein and procalcitonin concentrations in addition to symptoms and signs to predict pneumonia in patients presenting to primary care with acute cough: diagnostic study. BMJ 2013; 346:f2450.

41. Aabenhus R, Jensen JU, Jorgensen KJ, Hrobjartsson A, Bjerrum L. Biomarkers as point-of-care tests to guide prescription of antibiotics in patients with acute respiratory infections in primary care. Cochrane Database Syst Rev 2014;(11): CD010130.

42. Pollett S, Wood N, Boscardin WJ, et al. Validating the use of Google Trends to enhance pertussis surveillance in California. PLoS Curr 2015;7. 